\title{
NALOXONE INCREASES ELECTROPHYSIOLOGICAL MEASURES OF SELECTIVE INFORMATION PROCESSING IN HUMANS ${ }^{1}$
}

\author{
A. F. T. ARNSTEN, ${ }^{2}$ H. J. NEVILLE, ${ }^{*}$ S. A. HILLYARD, $\neq$ D. S. JANOWSKY, AND D. S. SEGAL ${ }^{3}$ \\ Departments of Psychiatry and $\ddagger$ Neuroscience, University of California, San Diego, La Jolla, California 92093 and $*$ The Salk \\ Institute, La Jolla, California 92037
}

Received November 29, 1983; Revised May 17, 1984; Accepted June 26, 1984

\begin{abstract}
The effects of the opiate antagonist naloxone on electrophysiological measures of human selective attention were examined utilizing a paradigm which dissociates selective information processing from any concurrent processes of general arousal that may be present. Subjects were injected with naloxone (2 mg, i.v.) or placebo prior to performing a three-channel selective listening task. The measure of selective attention was the difference between the auditory event-related potential (AERP) to a sequence of tones when they were attended and to the same sequence of tones when they were ignored. Typically, the AERP to attended channel tones is more negative, and this increased negativity is designated the attention effect. In this study, naloxone produced a significant augmentation of the AERP attention effect at frontal electrode sites, primarily by decreasing the negativity of AERPs to inattended tones. Naloxone had no effect on the AERPs from the undistracted and divided attention tasks or on the sensitivity of the AERP to a physical parameter of stimulus presentation, interstimulus interval. The effects of naloxone on selective attention appear to be independent of any alterations in arousal, as the drug had no effect on autonomic measures, reaction times, or auditory sensitivity, and the attention changes could be dissociated from any naloxone-induced alterations of mood. These data indicate that naloxone can have the specific effect of increasing AERP measures of selective information processing, thus suggesting a role for endogenous opioid peptides in the regulation of auditory selective attention in humans.
\end{abstract}

A long history of opiate usage in humans has facilitated our knowledge of the broad range of opiate effects. Opiates such as morphine produce not only analgesia and euphoria but also, at lower doses, the prominent effects of drowsiness, difficulty in mentation and concentration, apathy, occasional dysphoria, and lethargy (Jaffe and Martin, 1980). These morphine actions provide clues as to some of the possible functions of the endogenous opioid peptides.

Although much animal research on opioid function has concentrated on the analgesic role of these compounds, other studies demonstrate that opioids alter an animal's response to non-noxious stimuli as well. For example, morphine diminishes the acoustic startle response (Davis, 1980) and decreases reflexive responding to sensory stimulation (Goldfarb et al., 1978). We observed that morphine-treated rats spent less time

\footnotetext{
${ }^{1}$ We gratefully acknowledge Floyd E. Bloom and Lewis L. Judd for their consultation and financial support, Don Lawson for his technical expertise, and Greg Chesney, Jon Hansen, Marta Kutas, Regina Ciambrone, and Patricia Hermann for their assistance. This work has been reported elsewhere in abbreviated form (Arnsten et al., 1983) and was supported in part by United States Public Health Service Grants DA01994-06, MH-30914-06, NH-25594, and AA-03504. D. S. S. is the recipient of United States Public Health Service Career Scientist Award MH-70183-06.

${ }^{2}$ Present address: Section of Neuroanatomy, Yale University Medical School, 333 Cedar Street, New Haven, CT 06510.

${ }^{3}$ To whom correspondence should be addressed.
}

in contact with stimuli in a novel environment, and this reduction was blocked by the opiate antagonist naloxone (Arnsten et al., 1981). The administration of low doses of naloxone by itself significantly increased the time an animal spent per contact with stimuli (Arnsten and Segal, 1979; Arnsten et al., 1981). The stereoisomer (+)-naloxone, which has no actions at opiate receptors (lijima et al., 1978), had no effect on stimulus interaction (Arnsten et al., 1981). This series of results suggested that endogenous opioid systems were involved in the regulation of stimulus-directed behavior.

The prolonged stimulus contacts exhibited by naloxonetreated rats have been interpreted in terms of an opioid role in attention mechanisms (Arnsten et al., 1981). A similar interpretation was raised in regard to another study in which morphine-lreated rats made significantly more errors of commission while performing a detection task (Hernandez and Appel, 1979). Reports of human behavioral response also suggest that opioid peptides play a role in the regulation of attention. Anecdotal reports describe morphine impairing the ability to concentrate (Jaffe and Martin, 1980), whereas the opiate antagonist naltrexone has been shown to improve performance on a task sensitive to changes in attention and/or arousal (Gritz et al., 1976).

Findings from electrophysiological studies of humans given opiate drugs are also consonant with an opioid role in attention regulation. Naloxone increased, whereas morphine decreased, the amplitude of the $\mathrm{N} 1$ wave of the somatosensory eventrelated potential (Buchsbaum et al., 1977; Davis et al., 1982). 
The N1 wave is a negative peak, occurring approximately 100 to $150 \mathrm{msec}$ after stimulus onset, which varies in amplitude according to the degree of attention allotted to the evoking stimuli, the state of arousal of the subject, and the physical properties of the evoking stimuli (Naatanen, 1975; Desmedt and Robertson, 1977; Hillyard and Picton, 1979).

In the current study, we examined the effects of naloxone on the auditory event-related potential (AERP) in humans, utilizing a paradigm designed to dissociate the effects of attention from those of arousal and stimulus characteristics (Hillyard et al., 1973). In this paradigm, AERPs were recorded from subjects who listened selectively to one of three concurrently presented channels of rapidly occurring tone pips. One channel of tone pips was directed at the left ear, another at the right ear, and the third at the center of the head. Tones at each location were attended in turn, and subjects were instructed to detect occasional longer-duration target tones in the attended channel. This task resembles the classic "cocktail party" situation in which one voice is actively attended while others are ignored. The electrophysiological measure of selective attention was the difference between the AERP to the nontarget tone pips in a channel when it was attended and the AERP to the same sequence of nontarget tone pips when the channel was ignored. The amplitude of the AERP, including the N1 wave, was found to be substantially more negative when the tones were attended. This relative increase in the negativity elicited by attended channel tones has been designated the "attention effect." The amplitude of the attention effect has been loosely correlated with the subject's accuracy at detecting target stimuli in the attended channel(s) (Hink et al., 1977, 1978) and has been interpreted as an electrophysiological sign of the selective processing that is accorded to attended channel stimuli following an early (stimulus set) selection (e.g., Hillyard et al., 1973, Naatanen and Michie, 1979).

The experimental design described above provides a measure of selective attention distinct from the effects of the physical stimulus properties and changes in the state of arousal of the subject by employing the following procedures: (1) the exact same sequence of tones was repeated for cach attention condition, making the physical stimulus properties identical for attend and inattend AERPs; (2) the presentations of stimuli were randomized to prevent differential preparatory states (Naatanen, 1975); and (3) the order in which the different channcls were attended was controlled such that each condition occurred the same average length of time from the start of the session. In addition, the tones were presented rapidly to force selective attention to only one channel.

Previous research using variations of the above paradigm has shown that the early portion of the attention effect, which overlaps the $\mathrm{N} 1$ wave, is largest over the central scalp (at the vertex), whereas the later negativity is more frontally distributed (Hansen and Hillyard, 1980). In three-channel tasks such as the one employed in the present study, the early attention effect to the center channel tones was found to be smaller than the attention effects to the lateral channel tones (Schwent et al., 1976). This finding was interpreted as resulting from the poorer discriminability of the center channel, as there is less spatial separation between the central and the lateral channels.

If naloxone were to enhance this type of selective attention in humans, we would expect it to augment the AERP attention effect and, under appropriate conditions, to improve performance on the target detection task. This hypothesis was tested in the present study, in which subjects performed the threechannel selective attention task on each of the two sessions. They received naloxone on one day and placebo on the other according to a double blind, crossover design. In order to examine the specificity of naloxone's actions on the selective aspects of attention, subjects traditionally were tested for the following naloxone-induced alterations.

Undistracted and divided attention. In the undistracted or single-channel condition, a single sequence of tones was delivered to the center of the head. In the divided attention task, subjects attended to all three channels at once. The AERPs from the divided attention condition were used to create estimates of total attentional capacity. Attention researchers have proposed that the analysis of stimuli requires the allocation of attentional or processing resources from a limited pool (Kahneman, 1973; Norman and Bobrow, 1975). When the processing demands exceed the available resources, the allocation of capacity to one aspect of the task necessarily results in a reduced allocation to others. Results from two-channel AERP attention studies were found to be consistent with limited capacity theories of attention. The summated N1 amplitudes of the AERPs to the left and right channels remained constant whether attention was focused on one of the two channels or was divided between the two (Hink et al., 1977; Okita, 1979). This suggested that overall AERP negativity might be used as an estimate of total attentional capacity. In this study two measures of overall AERP negativity were examined as estimates of total attentional capacity: (1) the summated area of the attend and inattend waveforms across the three selective attention conditions, and (2) the summed areas of the AERPs to the left, right, and center channels from the divided attention condition.

$\Lambda E R P$ recovery functions. The amplitudes of several components of the AERP are sensitive to changes in interstimulus interval (ISI). The N1 (100 msec) and P2 (200 msec) peak amplitudes increase as a logarithmic "recovery function" of ISI (Nelson and Lassman, 1968). In the present study, subjects listened passively to a random sequence of tones of varying ISI to investigate whether naloxone might alter the N1/P2 peak amplitude recovery functions independent of any effects on attention.

Arousal and mood. Possible naloxone effects on arousal and mood were examined by looking for alterations of autonomic measures, reaction times, mood scale profiles, and global changes in attention measures, including the estimates of total attentional capacity.

The results from the above series of experiments support the hypothesis that naloxone has a specific effect of enhancing the selectivity of auditory attention.

\section{Materials and Methods}

Subjects. Subjects were young ( 20 to 28 years) adult male undergraduate and graduate students who were paid $\$ 100$ for their participation in the study. All subjects received physical examinations and demonstrated excellent health prior to acceptance in the study. Those who had abnormal hearing as determined by an audioradiogram or who were regular users of drugs such as marijuana or alcohol were omitted from the experiment. Subjects were instructed to refrain from alcohol intake $24 \mathrm{hr}$ before a test session, and those subjects who normally drank coffee were allowed one cup the morning of the experiment. The necessity of maintaining near identical conditions for the two sessions was impressed on all subjects. The general nature of the experiment was explained to them, but they were not informed of the specific hypotheses being tested.

Experimental protocol. Subjects arrived at the laboratory at 9:00 AM and responded to the mood scales while electrodes were affixed to their scalps. Subjects then were seated in an easy chair inside an electrically shielded, sound-attenuating chamber. Three channels of tone pips were delivered through earphones to the left ear, the right ear, or the center of the head (binaural). Auditory thresholds for the tone pips were taken for each ear, and the three channels were adjusted to be of equal intensity. In each of the three channels $10 \%$ of the tone pips were slightly longer in duration and were designated targets. A practice sequence of center tones only was given to familiarize the subject with the duration discrimination task. Subjects were instructed to press a button as quickly as possible each time they detected a target. Practice 
was continued until performance was highly accurate. The threechannel selective attention task then was demonstrated while the EEG was recorded. To reduce noise in the EEG record subjects were told to remain relaxed and keep still with their eyes open and to minimize eye movements and blinking.

Following the practice session the presiding physician took vital signs (pulse, blood pressure, and temperature). Subjects were then injected with naloxone ( $2 \mathrm{mg} / \mathrm{subject,} 0.03 \mathrm{mg} / \mathrm{kg}$, i.v. over $2 \mathrm{~min}$ ) on one experimental day and with a saline placebo on the other, according to a double blind, crossover design. Vital signs were taken again directly following the injection. Auditory thresholds were rechecked to detect changes in auditory sensitivity. Testing began approximately $10 \mathrm{~min}$ after the injection.

During the next 30 to $40 \mathrm{~min}$ subjects were tested on the selective attention, undistracted (single channel) attention, and divided attention tasks. There were three selective attention conditions: attend left channel, attend righ channel, and attend center channel. These tasks required the selective focusing of attention and the detection of targets in one channel when all three channels were present. In the singlechannel condition only the center channel was presented, such that subjects performed a simple duration discrimination task. In the divided attention task subjects were instructed to detect targets in all three channels at once. These five tasks were repeated twice, the second time in reverse order from the first such that all conditions occurred on the average the same length of time from injection. The order of condition presentation was varied between subjects according to a partial Latin Square Design. Subjects were allowed brief rests between tasks.

Following attentional testing subjects were instructed to relax and listen passively to a series of tones which were randomly delivered every $0.5,1.0$, or $3.0 \mathrm{sec}$ to the center of the head (binaural). This variation in ISI permitted examination of the recovery functions of the $\mathrm{N} 1$ and $\mathrm{P} 2$ peak amplitudes. Recovery function testing lasted approx imately $5 \mathrm{~min}$.

The exact protocol was repeated on the second session which occurred approximately 1 week later. However, following recovery function testing on the second session, an habituation experiment was performed in which the subject sat passively for 20 to $30 \mathrm{~min}$. The results of this experiment were not of sufficient interest to warrant presentation here. At the completion of both test sessions the electrodes were removed from the subject's scalp, blood pressure and pulse werc measured, and the subject once again responded to the mood scales. All testing occurred between 10:45 AM and 12:00 noon to minimize variability due to diurnal rhythms in opioid activity. Total testing time was approximately 50 to $70 \mathrm{~min}$ and thus occurred within the effective range of naloxone's actions.

Stimulus tapes. The stimuli for all attention tasks were $1000-\mathrm{Hz}$ tone pips with ISI randomized between 200 and $400 \mathrm{msec}$ (Gaussian distribution). In each channel $90 \%$ of the tone pips were $25 \mathrm{msec}$ in duration (standards) while the remaining $10 \%$ in each channel were $40 \mathrm{msec}$ in duration (targets). Tone intensities were $40 \mathrm{db}$ SL. The identical pattern of stimulation was used for each attention task and for each subject.

In the recovery function testing, stimuli were $800-\mathrm{Hz}$ tone pips of 25 msec duration. The ISIs were $0.5,1.0$, and $3.0 \mathrm{sec}$, and these occurred in random order. Stimulus intensity was $60 \mathrm{db} \mathrm{SL}$.

All sequences of tone pips were generated under computer control and were prerecorded along with coded trigger pulses on an audio tape recorder.

EEG recording and averaging. Electroencephalographic activity was recorded from frontal, central, and parietal midline sites $\left(\mathrm{F}_{z}, \mathrm{C}_{z}\right.$, and $\mathrm{P}_{\mathrm{z}}$ ), and from two lateral frontal sites (left, $\mathrm{F}_{3}$, right, $\mathrm{F}_{4}$ ), using $\mathrm{Ag}$ AgCl scalp electrodes (Fig. 1). Electrode resistances were kept below 2 kilohms. Electro-ocular activity was recorded from the lower left orbital ridge. All electrodes were referenced to linked mastoid electrodes.

Electroencephalographic and electro-ocular activities were amplified using Grass AC preamplifiers (time constant of $8 \mathrm{sec}$, bandpass 0.01 to $100 \mathrm{~Hz}$ ) and recorded on $\mathrm{FM}$ analogue tape along with coded trigger pulses for subsequent off-line anlalysis.

AERPs were digitized ( $1 \mathrm{msec} /$ point) and averaged separately for each task and for each class of stimulus using a PDP 11-45 computer. 'The averaging epoch began 50 (attention testing) or $100 \mathrm{msec}$ before stimulus onset and lasted for $1 \mathrm{sec}$ thereafter. Amplitudes were measured with respect to the mean base line voltãge during the prestimulus activity.

For the selective attention conditions, the attention effect was ob- served by averaging separately the AERPs to the left, right, or center standard tones under the condition when they were attended and the two conditions when they were inattended. The two "inattend" AERPs were averaged together. The attention-related negativity was calculated by subtracting the average inattend AERP from the attend AERP. This attention effect is represented by the stippled area in Figure 1. The area of this difference wave was measured from 0 to $200 \mathrm{msec}$ (early attention effect, which includes the N1 peak) and from 200 to $500 \mathrm{msec}$ (late attention effect) after stimulus onset. The same epochs were used in analyzing data from the single-channel and divided attention conditions and in calculating estimates of total attentional capacity.

In the recovery function condition, AFRPs were averaged separately for each of the three ISIs. The N1 and P2 peak amplitudes were calculated for each ISI (for N1, the largest negative peak occurred between 80 and $150 \mathrm{msec}$; for $\mathrm{P} 2$, the largest positive peak occurred between 150 and $200 \mathrm{msec}$. Peak rather than area amplitudes were used for direct comparison to previous studies of this kind (Nelson and Lassman, 1968).

Behavioral performance. In the five attention tasks, measures of both target detection and reaction time were taken. A button press within the 200 to $1000 \mathrm{msec}$ after the onset of each correct target was considered a hit, and all other button pressures were considered false alarms. Behavioral performance was assessed by modified signal detection analysis in which $d^{\prime}$ scores were used as indications of accuracy. In pilot studies the level of task difficulty was adjusted such that subjects performed at a $d^{\prime}$ of approximately 2.5 , thus permitting improvement in task performance.

Mood scales. Subjects rated themselves using the Profile of Mood States (POMS, McNair et al., 1971), the Beck Depression Inventory (Guy, 1976), and the Self-Report Activation-Inhibition Scale (Janowsky et al., 1973). The POMS includes the following scales: depression, anxiety, hostility, confusion, fatigue, vigor, elation, and friendliness. The Activation-Inhibition Scale rates cheerfulness, interactiveness, friendliness (activation), and lethargy/fatigue (inhibition). Preinjection assessment occurred on the subject's arrival at the laboratory and postinjection mood scales were administered at the very end of each session. Thus, on session I, mood scales were responded to approximately $5 \mathrm{~min}$ after the attention testing, whereas on session II mood scales were administered after the subjects had been seated passively for at least $20 \mathrm{~min}$. Subjects receiving naloxone during session I were analyzed separately from those receiving the drug during session II to account for this difference in protocol.

\section{Results}

Selective attention. Findings from placebo sessions replicated earlier reports; a three-way repeated measures analysis of variance (3-ANOVA-R) with factors of channels (left, right, and center), attention (attend or inattend), and electrodes revealed a highly significant main effect of attention for both the early and late epochs over all three channels (early, $F(1,9)=21.56$, $p<0.002$; late, $F(1,9)=22.65, p<0.001$; see Figs. $1^{*}$ and 2 ). The late attention effect had a more frontal distribution, as previously described by Hansen and Hillyard (1980) (Fig. 2; early attention by electrode interaction, $F(4,36)=1.74, p<$ 0.20 ; late attention by electrode interaction, $F(4,36)=7.04, p$ $<0.003,3$-ANOVA-R). Also consistent with previous reports (Schwent et al., 1976), the early attention effect to the center tones was smaller than those to the lateral channels at anterior electrode sites (Figs. 1 and 2; channel by electrode interaction, $F(8,72)=2.89, p<0.008,3$-ANOVA-R).

As seen in Figure 3, naloxone significantly augmented the late (200 to $500 \mathrm{msec})$ attention effect primarily at anterior electrode sites (drug by electrode interaction, $F(4,36)=4.57$, $p$ $<0.005,3$-ANOVA-R on attention effect data with factors of channels, drug, and electrodes). The early attention effect tended to be increased by naloxone, but this small effect at frontal electrode sites was not significant (drug by electrode interaction, $F(4,36)=1.46, p<0.30,3$-ANOVA-R).

The effect of naloxone on the late attention effect at the frontal sites was larger for the two lateral stimulus channels than for the center tones. To evaluate such channel-specific effects, separate 2-ANOVA-Rs were performed on the AERPs 


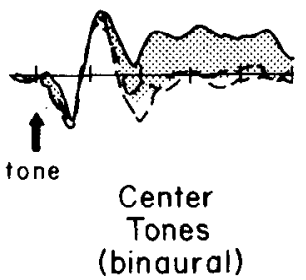

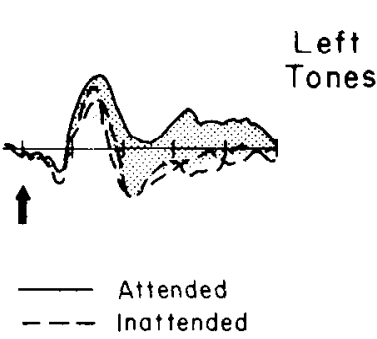

Average of 10 Subjects

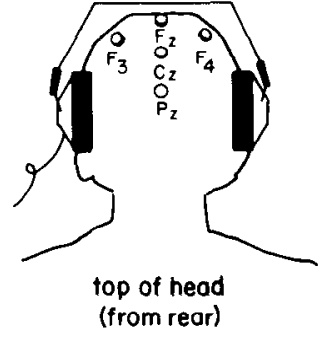

Placebo Condition
Right
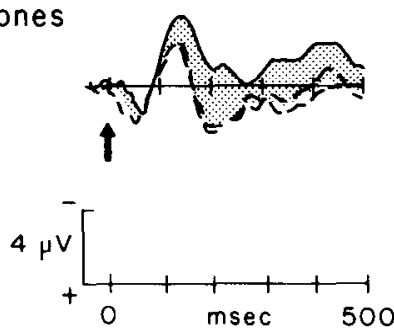

Figure 1. A diagram representing the perceived locations of the stimulus channels and the placements of the electrodes, and showing the grand average AERPs elicited by the left, center, or right standard tones following placebo administration, AERPs to attended channel tones (solid lines) and inattended channel tones (dashed lines) were recorded at the frontal midline electrode site $\left(F_{z}\right)$. The attention is illustrated by stippled shading.

\section{Left}
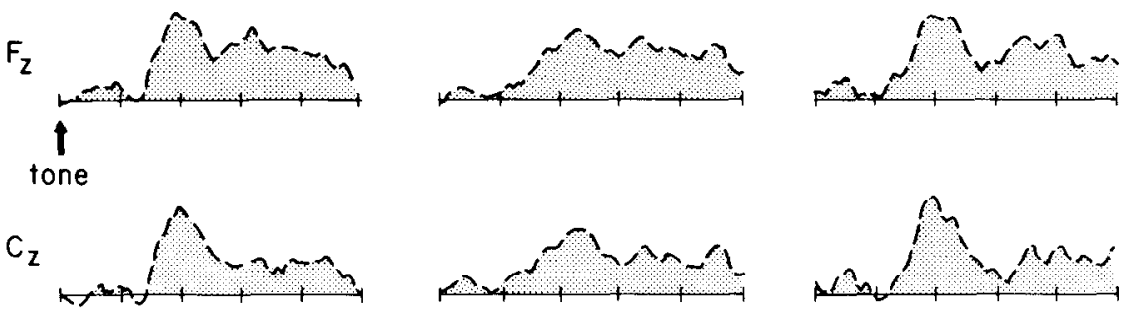

Right
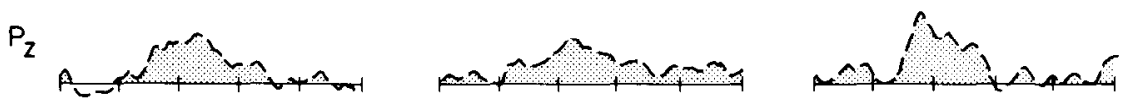

Average of 10 Subjects

Placebo Condition
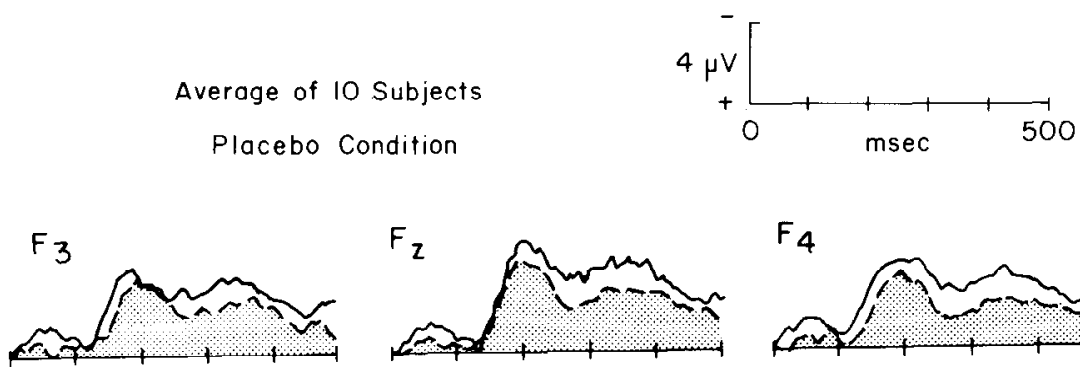

$\mathrm{F}_{4}$
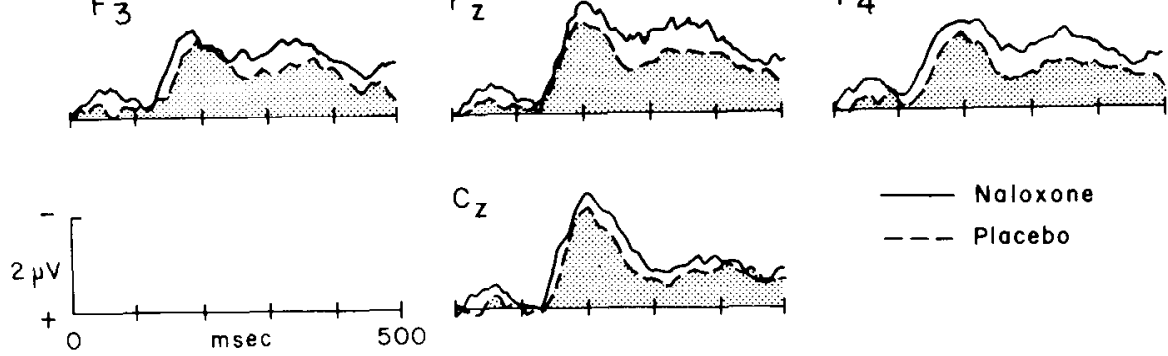

Noloxone

Placebo

$P_{Z}$

Average of 10 Subjects

Figure 2. The amplitude of the attention effect (stripped shading) to the left, center, or right standard tones at frontal, central, and parietal midline electrode sites following placebo administration.

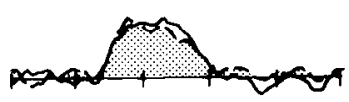

Figure 3. The effects of $2 \mathrm{mg}$ of naloxone on the amplitude of the attention effect averaged over left and right channel tones. Naloxone (solid lines) produced a significant augmentation of the attention effect at frontal electrode sites for the 200- to 500-msec epoch.

to the combined left and right channels and to the central channel (factors of drug and electrodes). Results from these analyses indicated that naloxone produced a significant augmentation of the late attention effect to left and right tones at frontal electrode sites (Table I; drug by electrode interaction: $F(4,36)=4.37, p<0.006,2$-ANOVA-R; simple main effects of drug: at $\mathrm{F}_{4}, F(1,9)=20.41, p<0.002$, at $\mathrm{F}_{\mathrm{z}}, F(1,9)=5.34, p<$ 0.05 , all other electrode sites not significant but had no signifi- 
TABLE I

The effects of naloxone on the late (200 to $500 \mathrm{msec}$ ) attention effect to the left + right or center tones

\begin{tabular}{cccccc}
\hline & \multicolumn{5}{c}{ Electrode } \\
\cline { 2 - 6 } & $\mathrm{F}_{3}$ & $\mathrm{~F}_{\mathrm{z}}$ & $\mathrm{F}_{4}$ & $\mathrm{C}_{\mathrm{z}}$ & $\mathrm{P}_{\mathrm{z}}$ \\
\hline Left + Right & & & & & \\
Naloxone & $-89.0^{a}$ & -121.0 & -96.1 & -82.6 & -20.4 \\
& $(15.3)^{b}$ & $(20.0)$ & $(18.7)$ & $(20.2)$ & $(17.9)$ \\
Placebo & -65.3 & -88.4 & -60.5 & -67.0 & -26.0 \\
& $(10.8)$ & $(15.8)$ & $(17.5)$ & $(15.8)$ & $(14.0)$ \\
Center & & & & & \\
Naloxone & -45.3 & -69.7 & -49.4 & -39.9 & 3.3 \\
& $(11.6)$ & $(14.1)$ & $(11.1)$ & $(13.1)$ & $(13.0)$ \\
Placebo & -59.6 & -87.9 & -70.1 & -73.4 & -38.3 \\
& $(21.1)$ & $(23.4)$ & $(23.6)$ & $(23.1)$ & $(22.2)$ \\
\hline
\end{tabular}

${ }^{a}$ Mean area amplitude.

${ }^{b}$ Numbers in parentheses are SEM.

TABLE II

The effects of naloxone on the attend and inattend waves to the left + right tones (200 to $500 \mathrm{msec}$ )

\begin{tabular}{lccccc}
\hline & \multicolumn{5}{c}{ Elẹctrode } \\
\cline { 2 - 6 } & $\mathrm{F}_{3}$ & $\mathrm{~F}_{\mathbf{z}}$ & $\mathrm{F}_{4}$ & $\mathrm{C}_{\mathrm{z}}$ & $\mathrm{P}_{\mathrm{z}}$ \\
\hline Attend & & & & & \\
Naloxone & $-45.3^{a}$ & -61.7 & -47.9 & -58.6 & -29.4 \\
& $(5.7)^{b}$ & $(9.9)$ & $(10.3)$ & $(12.1)$ & $(11.5)$ \\
Placebo & -32.8 & -47.1 & -34.6 & -45.9 & -24.0 \\
& $(9.5)$ & $(12.7)$ & $(12.5)$ & $(10.8)$ & $(7.4)$ \\
Inattend & & & & & \\
Naloxone & 31.3 & 42.3 & 31.9 & 14.8 & -9.3 \\
& $(8.0)$ & $(9.2)$ & $(6.8)$ & $(9.2)$ & $(7.0)$ \\
Placebo & 22.4 & 28.7 & 18.2 & 13.1 & -1.4 \\
& $(5.1)$ & $(6.3)$ & $(6.6)$ & $(9.5)$ & $(7.8)$ \\
\hline
\end{tabular}

${ }^{a}$ Mean area amplitude.

${ }^{b}$ Numbers in parentheses are SEM.

cant effects on the attention effect to the center tones (Table I; drug by electrode interaction, $F(4,36)=1.14, p>0.40,2$ ANOVA-R). Nine of the 10 subjects tested displayed a larger combined left and right ear attention effect at the $\mathbf{F}_{4}$ electrode site following naloxone administration.

The significant augmentation of the combined left and right ear attention effect resulted primarily from naloxone producing a positive shift of the inattend waves at frontal electrode sites only (Table II; drug by electrode interaction, $F(4,36)=5.68, p$ $<0.002$ ). Naloxone also produced a negative shift of the attend waves of all five electrode sites, but this did not reach statistical significance (Table II; drug, $F(1,9)=3.47, p>0.10,2$-ANOVA$\mathrm{R})$.

In contrast to pilot study results, ceiling effects prevented a sensitive measure of naloxone's effects on target detection (e.g., mean placebo $d^{\prime}=4.64$ ). However, naloxone did produce a small but significant improvement in behavioral performance for the "attend left" condition. Subjects who received naloxone in session II performed significantly better on the attend left task that session (naloxone, $d^{\prime}=4.91 \pm 0.84$; placebo, $d^{\prime}=$ $3.71 \pm 0.98, t_{\mathrm{dep}}=2.72, p<0.05$, two-tailed dependent $t$ test) This improvement was due to an interaction between naloxone and practice as demonstrated by the finding that subjects who received naloxone in session I did not perform significantly better in session II (naloxone, $d^{\prime}=4.49 \pm 0.54$; placebo, $d^{\prime}=$ $5.57 \pm 1.20, t_{\text {dep }}=1.08$, not significant). Naloxone had no consistent effect on the AERP response (P300 waves) to the target tones.
Undistracted and divided attention. As in the selective attention tasks, behavioral performance for the single-channel task during the placebo session was at maximum (mean $d^{\prime}=4.14$ \pm 0.41 ) and was unaffected by naloxone (mean $d^{\prime}=4.57 \pm$ 0.37). Scores for the divided attention task were lower than those for the selective and undistracted attention tasks (mean $d^{\prime}=2.57 \pm 0.18$ ), but they also were not altered by naloxone in either session (mean $d^{\prime}=2.58 \pm 0.19 ; t_{\mathrm{dep}}$, all $p$ values $>$ $0.10)$.

Naloxone had no effect on the AERPs to the standard tones in the undistracted (single channel) attention condition or on the two AERP estimates of attentional capacity, the summed waveforms from the divided and selective attention conditions (2-ANOVA-R, all $p$ values $>0.25)$. These results suggest that naloxone did not alter the amount of attentional or processing resources available to the subject.

$A E R P$ recovery functions. Results from placebo scssions demonstrated that the length of ISI had a highly significant effect on the amplitude of the N1 and P2 peaks, with longer ISIs being associated with target peak amplitudes (Fig. 4; N1 peak: ISI, $F(2,18)=26.96, p<0.00001 ; \mathrm{P} 2$ peak: ISI, $F(2,18)=17.99$, $p<0.0001,3$-ANOVA-R with factors of ISI $(0.5,1.0$, and 3.0 sec), drug (naloxone and placebo), and electrodes). The effects of ISI were largest at those scalp sites corresponding to the distribution of the $\mathrm{N} 1$ and $\mathrm{P} 2$ peaks largest effects of $\mathrm{N} 1$ peak at $\mathrm{F}_{\mathrm{z}}$ and $\mathrm{C}_{\mathrm{z}}$, largest effects of $\mathrm{P} 2$ peak at $\mathrm{C}_{\mathrm{z}}$ and $\mathrm{P}_{\mathrm{z}}$; ISI by electrode interactions: $F(8,72)=2.46$ and $7.04, p<0.02$ and $p$ $<0.00001$, respectively). Naloxone had no effect on the ampli-
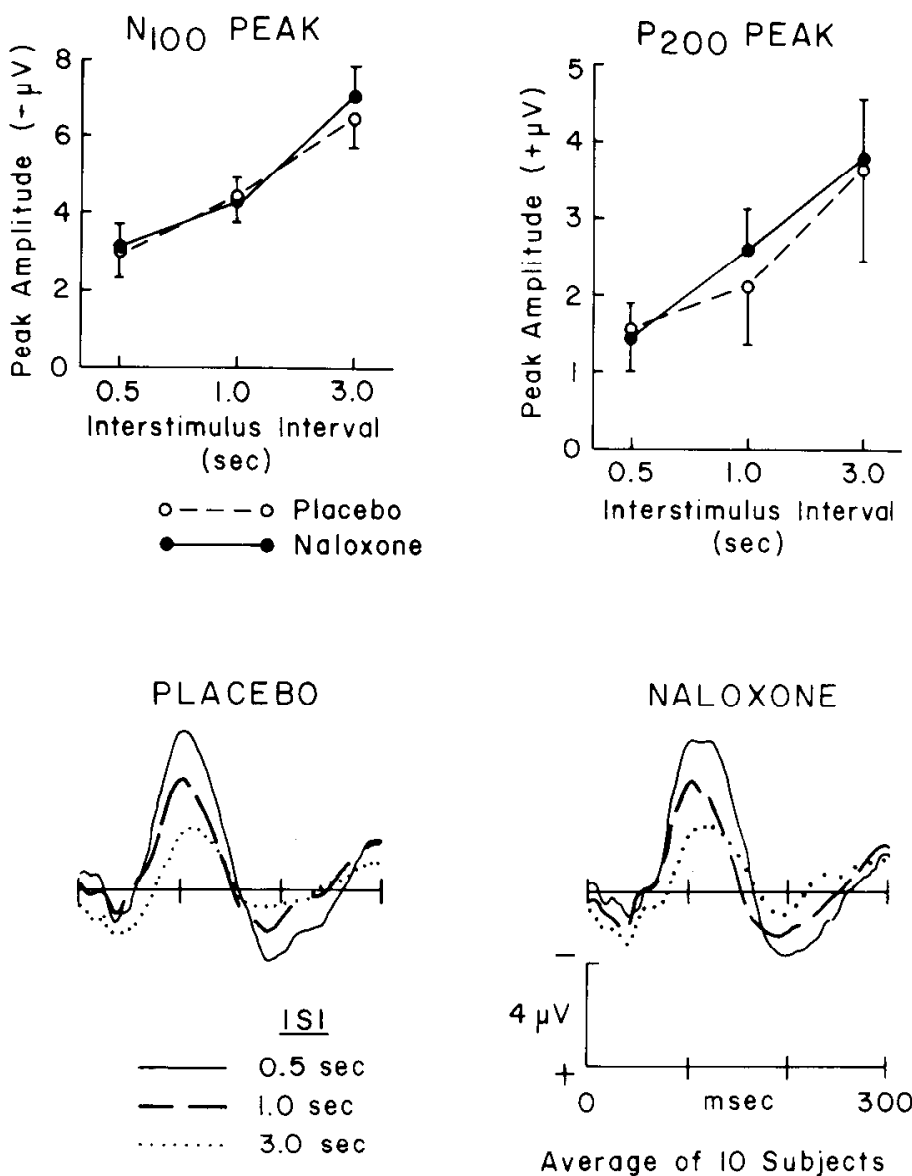

Figure 4. Mean peak amplitudes \pm SEM and grand average AERPs of the N1 (100 msec) and P2 (200 msec) waves evoked by stimuli occurring after one of three ISIs, $0.5,1.0$, or 3.0 sec. Peak amplitudes were increased by longer ISIs. Naloxone had no effect on this recovery function. 
tudes or the recovery functions of either the N1 or the P2 peaks (Fig. 4 ; all $p$ values $>0.40$.).

Arousal and mood. Naloxone had no effect on autonomic measures (blood pressure and pulse), auditory thresholds, or reaction times on any of the target detection attention tasks. As indicated above, the drug did not produce global changes of attention measures. Naloxone also had no significant effects on self-ratings on the mood scales, suggesting that the drug's effects on attention were not secondary to changes in arousal or mood.

Even if trends in the mood scale data are considered, naloxone's effects on mood or arousal cannot account for the drug's effects on attention. For example, subjects who received naloxone during session II and responded to the mood scales after the 20 -min passive condition tended to show positive correlations with the dysphoria scale (correlation between naloxone's effects on the AERP attention effect at $F_{z}$ and the dysphoria scale score: $r=+0.813, p<0.10, n=5$ ). In contrast, subjects who received naloxone during session I showed no correlation with the dysphoria scale $(r=0.000)$ but exhibited a tendency for a negative correlation with the confusion scale $(r=-0.822$, $p>0.10, n=5)$. As naloxone administration on either of the two sessions resulted in an augmentation of the attention effect, the drug's ability to alter attention thus can be dissociated from any potential effects on mood. Interestingly, previous research also suggests that naloxone's effects on mood may be situation dependent (Byck et al., 1982) and that dysphoria may result only under conditions in which the subject is passive (Jones and Herning, 1979).

Naloxone also tended to produce sedative effects (fatiguedrug by time interaction: $F(1,9)=3.89, p>0.10$; inhibitiondrug by time interaction: $F(1,9)=4.69, p>0.10,2$-ANOVA-R with factors of drug (naloxone and placebo) and time (pre- and postinjection), $n=10$ ). However, increased sedation cannot account for naloxone's effects on attention, as no fatiguerelated scale correlated with naloxone's alterations of the attention effect (fatigue, $r=+0.250$; inhibition, $r=-0.338, n=$ 10 ).

\section{Discussion}

Specificity of naloxone's effects. The pattern of results revealed by this series of experiments is consistent with naloxone having a specific action of enhancing the selectivity of auditory attention. Naloxone produced a clearly significant augmentation of the late attention effect but had no effect on measures of attention in the undistracted condition or on estimates of total attentional capacity. Naloxone did not alter the recovery properties of the $\mathrm{N} 1$ and $\mathrm{P} 2$ waves, nor did it have any effect on blood pressure, pulse rate, auditory thresholds, or reaction time. The effects of naloxone on attention-related negativity appear to be independent of any naloxone effects on mood. This specificity of naloxone's effects makes it unlikely that the increase in attentional selectivity was mediated by an overall change in arousal level, which would be expected to produce more widespread changes in these electrophysiological, behavioral, and autonomic indices. This view is supported by the mood scale data in which no arousal-related scale (e.g., vigor, fatigue, inhibition) correlated with naloxone's actions on the attention effect.

Recent clinical studies of Alzheimer's patients have also found that naloxone can improve attention (Reisberg et al., $1983 \mathrm{a}, \mathrm{b})$. Naloxone significantly improved scores on the "concentration" as well as the "memory" and "orientation" axes of the Brief Clinical Rating Scale (Reisberg et al., 1982). Although unexpected ceiling effects impaired our ability to examine naloxone's effects on behavioral performance, the clinical findings with Alzheimer's patients are encouraging and suggest that naloxone can augment behavioral as well as electrophysiological measures of selective attention.

The augmentation of the attention effect produced by naloxone resulted more from a positive shift in the inattend waves at frontal electrode sites than from a negative shift in the attend waveform. This suggests that, under the conditions of this study, naloxone improved selective attention primarily by allowing a more effective rejection of irrelevant stimuli. Whether this increase in selectivity of attention was due to a narrowing of the focus of attention at any one time or to a decrease in attentional lability (distractability) cannot be determined from these results. However, as naloxone did not alter the attention effect to the less discernable center tones, it is possible that naloxone facilitates the suppression of irrelevant stimuli only when those stimuli can be readily discriminated from the relevant tones. The low naloxone dose utilized in this study and our previous pharmacological studies in rats (Arnsten and Segal, 1979; Arnsten et al., 1981) indicate that these naloxone effects on selective attention may result from actions at opioid receptors rather than nonspecific properties of the drug. These data thus suggest a role for endogenous opioid systems in the regulation of selective attention in humans.

Possible neural mechanisms. The pattern of naloxone binding in the primate cortex has been interpreted as suggesting an opioid role in selective attention (M. E. Lewis et al., 1981; Wise and Herkenham, 1982). Naloxone binding is lowest in primate sensory areas and increases along a gradient in relation to the degree of processing such that polysensory association areas demonstrate the densest naloxone binding (M. E. Lewis et al., 1981). The prefrontal cortex is rich in naloxone binding (Kuhar et al., 1973; Divoc et al., 1981; M. E. Lewis et al., 1981) and, in the rodent, has been shown to contain intrinsic enkephalinimmunoreactive neurons (McGinty et al., 1982). A prefrontal cortical role in attention regulation has been indicated by the increased distractability exhibited by frontally lesioned humans (Damasio, 1979) and animals (Grueniger and Pridham, 1969) and by the supposed role of these brain regions in suppressing responses to irrelevant stimuli (Bartus and Levere, 1977; Wilcott, 1977). Regional blood flow studies in humans indicate that the right medial prefrontal cortex in particular is active when the subject is performing a selective attention task (Roland, 1982). It has been suggested that the prefrontal cortex modulates event-related potential attention-related negativity (Hillyard and Picton, 1979). In line with this proposal, patients with unilateral prefrontal cortical lesions exhibited significantly smaller attention effects (Knight et al., 1981) while having normal N1/P2 recovery functions (Knight et al., 1980). The decrease in the attention effect in the patients of Knight et al. $(1980,1981)$ appeared to result mostly from an inability to suppress negativity to inattended stimuli contralateral to the lesion, particularly for those patients with right frontal lesions. In this context it is of interest that naloxone similarly had no effect on the $\mathrm{N} 1 / \mathrm{P} 2$ recovery functions and increased the attention effect largely by enhancing the suppression of negativity to inattended tones. Furthermore, these naloxone actions occurred exclusively at frontal electrode sites, with the most consistent effects being observed at the right frontal $\left(\mathrm{F}_{4}\right)$ electrode site. Thus, naloxone's effects may involve a facilitation of certain prefrontal cortical functions.

Previous research with rats has shown that the expression of the naloxone response requires the presence of an intact noradrenergic (NE) system. For example, we observed that naloxone did not increase stimulus-directed behavior in rats with extensive forebrain NE depletions (Arnsten et al., 1981). Similarly, naluxone's facilitory effects on memory were blocked by the $\beta$-receptor antagonist propanolol (Izquierdo and Graudenz, 1980). These results are consistent with the inhibitory effects of opioids on NE transmission (Korf et al., 1974; Bird and 
Kuhar, 1977; Arbilla and Langer, 1978; Llorens et al., 1978; Strahlendorf et al., 1980). It is conceivable that similar opioid/ $\mathrm{NE}$ interactions exist in humans and that naloxone's actions on the attention effect at least partially result from facilitation of NE transmission. Several behavioral studies report results consistent with the interpretation that forebrain noradrenaline is necessary for an animal to ignore distracting stimuli (Roberts et al., 1975; Oke and Adams, 1978; Mason and Iversen, 1979; Lorden et al., 1980). Consonant with these behavioral results are the electrophysiological findings which demonstrate that noradrenaline enhances the signal-to-noise ratio of responding of postsynaptic cells. Generally, the background cell activity is suppressed whereas the responses to cell-appropriate stimuli are highlighted (Foote et al., 1975; Segal and Bloom, 1976; Waterhouse et al., 1980). This NE effect resembles naloxone's alterations of the AERPs to inattended and attended tones.

Clinical relevance. In human studies, the exposure to stressors can produce an inability to ignore irrelevant stimuli (Broadbent, 1971), particularly during the performance of complex tasks (Hockey, 1970). The stress associated with high levels of arousal is thought to narrow attentional focus and increase distractability (Kahneman, 1973). Many reports indicate that endogenous opioid systems can be activated under conditions of stress. Exposure to stressors can promote the release of opioid peptides from the pituitary (Guillemin et al., 1977; Mueller, 1981), adrenal medulla (Viveros et al., 1979), and brain (Akil et al., 1976), and can produce opiate-like, naloxonereversible effects on behavior in animals $(\mathrm{J}$. W. Lewis et al., 1981) and humans (Frid et al., 1979; Willer et al., 1981). We have observed that rats exposed to stressors exhibit morphinelike reductions in stimulus-directed behavior which are blocked by very low doses of naloxone (Arnsten et al., 1984). It is possible that stress-induced alterations of human selective attention similarly involve an opioid mechanism.

The results presented in this paper suggest that naloxone or other opiate antagonists may be useful in the treatment of attention disorders. For example, children with attention deficit disorder (hyperkinesis) exhibit poor selective attention (American Psychiatric Association, 1980) and smaller AERP attention effects (Zambelli et al., 1977) than do age-matched controls. If the naloxone-induced alterations of the AERPs in adult males are indicative of a general improvement in selective information processing, it is possible that opiate antagonists may be effective in treating patients suffering from selective attention deficits.

\section{References}

Akil, H., J. Madden, R. L. Patrick, and J. D. Barchas (1976) Stressinduced increase in endogenous opiate peptides: Concurrent analgesia and its partial reversal by naloxone. In Opiates and Endogenous Opioid Peptides, H. W. Kosterlitz, ed., pp. 63-70, Elsevier-North Holland Publishing Co., Amsterdam.

American Psychiatric Association (1980) Diagnostic and Statistical Manual of Mental Disorders, Ed. 3, J. B. W. Williams, text ed., American Psychiatric Association, Washington, D. C.

Arbilla, S., and S. Z. Langer (1978) Morphine and $\beta$-endorphin inhibit release of noradrenaline from cerebral cortex but not of dopamine from rat striatum. Nature 271: 559-560.

Arnsten, A. T., and D. S. Segal (1979) Naloxone alters locomotion and interaction with environmental stimuli. Life Sci. 25: 1035-1042.

Arnsten, A. F. T., D. S. Segal, S. E. Loughlin, and D. C. S. Roberts (1981) Evidence for an interaction of opioid and noradrenergic locus coeruleus systems in the regulation of environmental stimulus-directed behavior. Brain Res. 222: 351-363.

Arnsten, A. F. T., D. S. Segal, D. S. Janowsky, L. L. Judd, S. A. Hillyard, H. E. Neville, and F. E. Bloom (1983) Electrophysiological signs of selective attention in man. Nature 304: 725-727.

Arnsten, A. F. T., C. Berridge, and D. S. Segal (1984) Stress produces opioid-like effects on investigatory and locomotor behaviors. Pharmacol. Biochem. Behav., in press.
Bartus, R. T., and T. E. Levere (1977) Frontal decortication in rhesus monkeys: A test of the interference hypothesis. Brain Res. 119: 233248.

Bird, S. J., and M. J. Kuhar (1977) Iontophoretic application of opiates in the locus coeruleus. Brain Res. 122: 523-533.

Broadbent, D. (1971) Decision and Stress, Academic Press, Inc., New York.

Buchsbaum, M. S., G. C. Davis, and W. E. Bunney, Jr. (1977) Naloxone alters pain perception and somatosensory evoked potentials in normal subjects. Nature 270: 620-622.

Byck, R., A. Ruskis, J. Ungerer, and P. Jatlow (1982) Naloxone potentiates cocaine's effect in man. Psychopharmacol. Bull. 18: 214-215.

Damasio, A. (1979) The frontal lobes. In Clinical Neuropsychology, K. M. Heilman and E. Valenstein, eds., pp. 360-412, Oxford University Press, New York.

Davis, G. C., M. S. Buchsbaum, and W. E. Bunney (1982) The endorphins. In Neural Peptides and Neuronal Communications, E. Costa and M. Trabucchi, eds., pp. 473-487, Raven Press, New York.

Davis, M. (1980) Neurochemical modulation of sensory-motor reactivity: Acoustic and startle reflexes. Neurosci. Biobehav. Res. 4: 241263

Desmedt, J. E., and D. Robertson (1977) Differential enhancement of early and late components of the cerebral somatosensory evoked potentials during forced-paced cognitive tasks in man. J. Physiol. (Lond.) 271: 761-782.

Divoc, I., C. Braestrup, and M. Nielsen (1981) Spiroperidol, naloxone, diazepam, and QNB binding in the monkey cerebral cortex. Brain Res. Bull. 7: 469-477.

Foote, S. L., R. Freedman, and A. P. Oliver (1975) Effect of putative neurotransmitters on neuronal activity in monkey and auditory cortex. Brain Res., 86: 229-242.

Frid, M., G. Singer, and C. Rana (1979) Interactions between personal expectations and naloxone: Effects on tolerance to ischemic pain. Psychopharmacology 65: 225-231.

Goldfarb, J., E. I. Kaplan, and H. R. Jenkins (1978) Interaction of morphine and naloxone in acute spinal cats. Neuropharmacology 15: $569-575$.

Gritz, E. R., S. M. Shiffman, M. E. Jarvik, J. Schlesinger, and V. C. Charuvastra (1976) Naltrexone: Physiological and psychological effects of single doses. Clin. Pharmacol. Ther. 19: 773-776.

Grueniger, W. E., and K. II. Pridham (1969) Effects of spatial and nonspatial distractors on performance latency of monkeys with frontal lesions. J. Comp. Physiol. Psychol. 68: 203-209.

Guillemin, R., T. M. Vargo, J. Rossier, S. Minick, N. Ling, C. Rivier, W. Vale, and F. E. Bloom (1977) Beta-endorphin and adrenocorticotropin are secreted concomitantly by the pituitary gland Science 197: $1367-1369$

Guy, W. (1976) ECDEU Assessment Manual for Psychopharmacology, DHEW Publication No. 76-338, Superintendent of Documents, U. S. Government Printing Office, Washington, D. C.

Hansen, J., and S. A. Hillyard (1980) Endogenous brain potentials associated with selective auditory attention. Electroencephalogr. Clin. Neurophysiol. 49: 277-290.

Hernandez, L. L., and J. B. Appel (1979) An analysis of some perceptual effects of morphine, chlorpromazine and LSD. Psychopharmacology 60: $125-130$.

Hillyard, S., and T. W. Picton (1979) Event-related brain potentials and selective information processing in man. In Progress in Clinical Neurophysiology, J. Desmedt, ed., pp. 1-50, S. Karger AG, Basel.

Hillyard, S. A., R. F. Hink, V. L. Schwent, and T. W. Picton (1973) Electrophysiological signs of selective attention in the human brain. Science 182: 177-180.

Hink, R. F., S. Van Voorhis, S. A. Hillyard, and T. S. Smith (1977) The division of attention and the human auditory evoked potential. Neuropsychologia 15: 597-605.

Hink, S., S. A. Hillyard, and P. J. Benson (1978) Event-related potentials and selective attention to acoustic and phonetic cues. Biol. Psychol. 6: 1-16.

Hockey, G. R. J. (1970) Effect of loud noise on attentional selectivity. Q. J. Exp. Psychol. 22: 28-36.

Iijima, I., J. Minamikawa, A. E. Jacobson, A. Brossi, K. C. Rice, and W. Klee (1978) Studies in the $(+)$-morphinian series. 5.1 Synthesis and biological properties of $(+)$-naloxone. J. Med. Chem. 21: 398400.

Izquierdo, I., and M. Graudenz (1980) Memory facilitation by naloxone 
is due to release of dopaminergic and $\beta$-adrenergic systems from tonic inhibition. Psychopharmacology 67: 265-268.

Jaffe, J. H., and J. R. Martin (1980) Opioid analgesics and antagonists In The Pharmacological Basis of Therapeutics, Ed. 6, A. G. Gilman, L. S. Goodman, and A. Gilman, eds., p. 494, Macmillan, New York.

Janowsky, D. S., M. K. El-Yousef, J. M. Davis, and H. J. Sedkerke (1973) Parasympathetic suppression of manic symptoms by physostigmine. Arch. Gen. Psychiatry 28: 542-547.

Jones, R. T., and R. I. Herning (1979) Naloxone-induced mood and physiologic changes in normal volunteers. In Endorphins in Mental Health Research, E. Usdin, ed., pp. 484-491, Macmillan, New York.

Kahneman, D. (1973) Attention and Effort, Prentice-Hall, Englewood Cliffs, NJ.

Knight, R., S. A. Hillyard, D. L. Woods, and H. Neville (1980) The effects of frontal and temporal-parietal lesions on the auditory evoked potential in man. Electruencephalogr. Clin. Neurophysiol. 50: 112-124.

Knight, R., S. A. Hillyard, D. L. Woods, and H. Neville (1981) The effects of frontal cortex lesions on event-related potentials during auditory selective attention. Electroencephalogr. Clin. Neurophysiol. 52: 571-582.

Korf, J., B. S. Bunney, and G. K. Aghajanian (1974) Noradrenergic neurons: Morphine inhibition of spontaneous activity. Eur. J. Pharamcol. 25: 165-169.

Kuhar, M. J., C. B. Pert, and S. H. Snyder (1973) Regional distribution of opiate receptor binding in monkey and human brain. Nature 245 : $147-450$.

Lewis, J. W., J. E. Sherman, and J. C. Liebeskind (1981) Opioid and non-opioid stress analgesia: Assessment of tolerance and cross-tolerance with morphine. J. Neurosci. 1: 358-363.

Lewis, M. E., M. Mishkin, G. Bragin, R. M. Brown, C. B. Pert, and A. Pert (1981) Opiate receptor gradients in monkey cerebral cortex: Correspondence with sensory processing hierarchies. Science 211: 1166-1169.

Llorens, C., M. P. Martres, M. Baudry, and J. C. Schwartz (1978) Hypersensitivity to noradrenaline in cortex after chronic morphine: Relevance to tolerance and dependence. Nature 274: 603-605.

Lorden, J. F., E. J. Rickett, R. Dawson, and M. A. Pelleymounter (1980) Forebrain norepinephrine and the selective processing of information. Brain Res. 190: 569-573.

Mason, S. T., and S. D. Iversen (1979) Theories of the dorsal bundle extinction effect. Brain Res. Rev. 1: 107-137.

McGinty, J. F., D. van der Kooy, L. Y. Koda, and F. E. Bloom (1982) Enkephalin immunoreactive cells in frontal olfactory and limbic cortex. Anat. Rec. 202: 125a.

McNair, D., M. Lorr, and L. Droppleman (1971) EITS Manual: The Profile of Mood States, Educational and Industrial Testing Service, San Diego.

Mueller, G. P. (1981) $\beta$-Endorphin immunoreactivity in rat plasma: Variations in response to different physical stimuli. Soc. Neurosci. Abstr. 7: 134.

Naatanen, R. (1975) Selective attention and evoked potentials in humans-A critical review. Biol. Psychol. 2: 237-307.

Naatanen, R., and M. T. Michie (1979) Early selective attention effects and the evoked potential. A critical review and reinterpretation. Biol. Psychol. 8: 81-136.

Nelson, D. A., and F. M. Lassman (1968) Effects of intersignal interval on the human auditory evoked response. J. Acoust. Soc. Am. 44: $1529-1532$.

Norman, D. A., and D. G. Bobrow (1975) On data-limited and resources-limited processes. Cognitive Psychol. 7: 44-64.

Oke, A. F., and R. N. Adams (1978) Selective attention dysfunctions in adult rats neonatally treated with 6 -hydroxydopamine. Pharmacol. Biochem. Behav. 9: 429-432.

Okita, T. (1979) Event-related potentials and selective attention to auditory stimuli varying in pitch and localization. Biol. Psychol. 9: 271-284.

Parasuraman, R. (1978) Auditory evoked potentials and divided attention. Psychophysiology 15: 460-465.

Reisberg, B., S. H. Ferris, M. J. DeLeon, and T. Crook (1982) The global determination scale for assessment of primary degenerative dementia. Am. J. Psychiatry 139: 1136-1139.

Reisberg, B., S. H. Ferris, R. Anand, P. Mir, M. J. DeLeon, and E Roberts (1983a) Naloxone effects on primary degenerative dementia (PDD). Psychopharmacol. Bull. 19: 44-47.

Reisberg, B., S. H. Ferris, R. Anand, M. Pervez, V. Geibel, M. L. DeLeon, and E. Roberts (1983b) Effects of naloxone in senile dementia: A doubleblind trial. N. Engl. J. Med. 308: 721-722.

Roberts, D. S. C., M. T. C. Price, and H. C. Fibiger (1975) The dorsal tegmental noradrenergic projection: An analysis of its role in maze learning. J. Comp. Physiol. Psychol. 90: 363-372.

Roland, P. E. (1982) Cortical regulation of selective attention in man A regional cerebral blood flow study. J. Neurophysiol. 48: 1059-1078.

Schwent, V. L., E. Snyder, and S. A. Hillyard (1976) Auditory evoked potentials during multichannel selective listening: Role of pitch and localization cues. J. Exp. Psychol. 2: 313-325.

Segal, M., and F. E. Bloom (1976) The action of norepinephrine in the rat hippocampus. IV. The effects of locus coeruleus stimulation on evoked hippocampal unit activity. Brain Res. 107: 513-525.

Strahlendorf, H. K., J. C. Strahlendorf, and C. D. Barnes (1980) Endorphin-mediated inhibition of locus coeruleus neurons. Brain Res. 191: 284-288.

Viveros, O. H., E. J. Dilberto, E. Hazum, and K. Chang (1979) Opiatelike materials in the adrenal medulla: Evidence for storage and secretion with catecholamines. Mol. Pharmacol. 16: 1101-1108.

Waterhouse, B. D., H. C. Moises, and D. J. Woodward (1980) Locus coeruleus stimulation potentiates somatosensory cortical neuronal responses to afferent synaptic inputs. Soc. Neurosci. Abstr. 6: 448.

Wilcott, R. C. (1977) Electrical stimulation of the prefrontal cortex and delayed response in the cat. Neuropsychologia 15: 115-121.

Willer, J. C., H. Dehen, and J. Cambier (1981) Stress-induced analgesia in humans: Endogenous opioids and naloxone-reversible depression of pain reflexes. Science 212: 689-691.

Wise, S. P., and M. Herkenham (1982) Opiate receptor distribution in the cerebral cortex of the rhesus monkeys. Science 218: 387-389.

Zambelli, A. S., J. S. Stamm, S. Maitinsky, and D. Loiselle (1977) Auditory evoked potentials and selective attention in former hyperactive adolescent boys. Am. J. Psychiatry 134: 742-747. 